\title{
Selective IgA deficiency - does partial mean negligible?
}

\author{
Katarzyna Tąpolska-Jóźwiak, Aleksandra Szczawińska-Popłonyk \\ Department of Paediatric Pneumonology, Allergology, and Clinical Immunology, Poznan University of Medical Sciences, \\ Poznan, Poland
}

\section{ABSTRACT}

Introduction: Selective immunoglobulin A deficiency (SIgAD) is the most common primary immunodeficiency in the Caucasian population. The current definition describes SIgAD as serum IgA level below $0.07 \mathrm{~g} / \mathrm{l}$ in individuals of four years of age or older with normal immunoglobulin $G$ and M levels, in whom other causes of hypogammaglobulinaemia have been excluded. Most patients with SIgAD are asymptomatic, but some of them suffer from recurrent infections, primarily involving the respiratory system, allergies, autoimmune disorders, and malignancies. In contrast to "absolute" SIgAD, the clinical relevance of partial SIgAD has not yet been determined.

Aim of the study: To compare the clinical course of absolute and partial SIgAD in children.

Material and methods: The study was conducted through a retrospective review of the medical records of 25 children with absolute and 33 children with partial SIgAD, aged between 4 and 18 years, for the occurrence of recurrent respiratory, urinary, and gastrointestinal tract infections, as well as allergy, autoimmunity, and malignancies.

Results: Respiratory tract infections were the most common clinical manifestation in both variants of SIgAD and affected nearly two-thirds of patients with absolute and about three-quarters with partial SIgAD. Apart from autoimmunity in total, which occurred more often in children with absolute than partial SIgAD (44\% vs. $12.12 \%, p=0.006$ ), no statistically significant differences were found between the incidence of the analysed conditions in both groups.

Conclusions: Due to the similar clinical course of both variants of SIgAD, all symptomatic patients should receive specialist care and undergo systematic follow-up visits. In children with autoimmune diseases basic immunology screening should be considered.

KEY WORDS:

immunoglobulin A, primary immunodeficiencies, recurrent infections, allergy, autoimmunity.

\section{INTRODUCTION}

Selective immunoglobulin A deficiency (SIgAD) is a humoral primary immunodeficiency (PID), with an estimated prevalence of 1 in 600, which makes it the most common immunodeficiency in the Caucasian population [1]. However, the frequency obtained in adults should not be extrapolated to children, because IgA reaches adult levels in early teens - according to the literature, the prevalence in children ranges from 1 in 90 in the Netherlands to 1 in 2095 in Tunisia $[2,3]$. The current definition describes SIgAD as serum IgA level below $0.07 \mathrm{~g} / \mathrm{l}$ in individuals of four years of age or older with normal immunoglobulin $\mathrm{G}(\mathrm{IgG})$ and immunoglobulin M (IgM) levels,

\section{ADDRESS FOR CORRESPONDENCE:}

Katarzyna Tąpolska-Jóźwiak, Department of Paediatric Pneumonology, Allergology, and Clinical Immunology, Poznan University of Medical Sciences, 27/33 Szpitalna St., 60-572 Poznan, Poland, ORCID: 0000-0002-7448-3370, e-mail: ktapolska@wp.pl 
in whom other causes of hypogammaglobulinaemia have been excluded. Most patients with SIgAD remain asymptomatic; nevertheless, some of them suffer from recurrent infections, primarily involving the respiratory system, allergies, autoimmune disorders, and malignancies [2, 4].

In contrast to "absolute" SIgAD (aSIgAD), "partial" SIgAD (pSIgAD) is characterised by IgA level 2SD below normal for age and is commonly considered as a mild variant of SIgAD, not requiring specialist care [5]. Recent studies reviewed the potential risk of significant symptoms in pSIgAD; however, the results obtained were not consistent. Issues regarding the standard of care for patients with pSIgAD remain unresolved; it is discussed whether they need systematic control by an immunology specialist, as well as whether their immunodeficiency is associated with potential health complications.

The aim of the study was to compare the clinical picture of both variants of SIgAD in symptomatic children to determine their relevance in everyday medical practice.

\section{MATERIAL AND METHODS}

\section{MATERIAL}

The study was conducted through a retrospective review of the medical records from the department of paediatric pneumonology, allergology, and clinical immunology, from June to December 2019. Patients with aSIgAD and pSIgAD, aged between 4 and 18 years, were identified in the course of differential diagnosis of recurrent infections, allergies, and/or autoimmune diseases. In creating the study group, we used the above definition and reference values for age, developed for the population of healthy children in the central clinical hospital laboratory (Table 1). Immunoglobulin levels were determined in the blood venous immunoturbidimetric method (Beckman Coulter, USA).

The initial sample consisted of 27 children with IgA serum $<0.07 \mathrm{~g} / \mathrm{l}$, but we excluded two individuals with aSIgAD diagnosed after bone marrow transplantation undergoing oncological treatment, due to its possible impact on test results and presented symptoms. Finally, 25 patients met the criteria of aSIgAD and 33 of pSIgAD; the groups did not differ significantly in age. Table 2 presents the clinical characteristics of both groups.

TABLE 1. Normal serum levels of immunoglobulins ( $g / l)$

\begin{tabular}{|l|c|c|c|}
\hline Age in years & $\lg \mathrm{G}$ & $\lg \mathrm{A}$ & $\lg \mathrm{M}$ \\
\hline $4-5$ & $5.40-14.20$ & $0.52-2.20$ & $0.40-2.00$ \\
\hline $6-7$ & $5.70-14.10$ & $0.65-2.40$ & $0.55-2.10$ \\
\hline $8-9$ & $7.30-14.10$ & $1.08-2.00$ & $0.55-1.75$ \\
\hline $10-11$ & $7.30-13.50$ & $0.91-2.55$ & $0.66-1.55$ \\
\hline $12-13$ & $7.70-15.10$ & $1.08-3.25$ & $0.70-1.50$ \\
\hline$>13$ & $7.00-16.00$ & $0.70-4.00$ & $0.40-2.40$ \\
\hline
\end{tabular}

\section{METHODS}

In order to compare both groups in terms of occurrence of clinically significant symptoms, we analysed the patients' medical history, paying special attention to: (1) recurrent infections, taking into consideration infections of upper respiratory tract (rhinosinusitis, laryngitis), infections of lower respiratory tract (bronchitis, pneumonia), and otitis media, as well as gastrointestinal infections, urinary tract infections, and deep-seated infections including sepsis; (2) allergy, comprising food allergy, atopic dermatitis, asthma, allergic rhinitis, and urticaria; (3) autoimmune disorders with an indication of the frequency of individual diseases; (4) malignancies; and (5) family history of PID.

\section{STATISTICAL ANALYSIS}

Differences in the distribution of selected characteristics were tested using Pearson's $\chi^{2}$ test and Fischer's exact test in cases when the assumptions for the $\chi^{2}$ test were not met. We used a significance cut-off of $p=0.05$ for each test. All analyses were performed with Statistica version 13.3.

The study was approved by the local institutional Bioethical Committee.

\section{RESULTS}

The results of the study are summarised in Table 2. Respiratory tract infections were the most common clinical manifestation both in aSIgAD and pSIgAD, and affected nearly two-thirds of patients with aSIgAD and about three-quarters with pSIgAD. It is apparent that apart from the autoimmunity in total, no statistically significant differences were found between the incidence of the analysed conditions in both groups.

\section{DISCUSSION}

Recurrent respiratory tract infections remain the most common symptom of PID, as well as the most common reason for referring a child to the department of immunology. Lower respiratory tract infections are more specific for PID, but in children, upper respiratory tract infections are also of clinical importance [6-9]. Recurrent pneumonia occurs in $14-23 \%$ of symptomatic IgA-deficient children, and, according to the literature, up to $15-30 \%$ of them will develop bronchiectasis [9-11]. On the other hand, repetitive upper respiratory tract infections increase the risk of cochlear sensorineural hearing loss [12]. Infections in SIgAD are typically caused by extracellular encapsulated bacteria (e.g. Streptococcus pneumonia, Haemophilus influenzae) and occur more often in patients with concomitant IgG subclass deficiency [2, 13]. In SIgAD the number of IgM-bearing B-cells increases 
TABLE 2. General characteristics and the comparison of clinical manifestations of patients with aSlgAD and pSIgAD

\begin{tabular}{|c|c|c|c|c|c|}
\hline Parameter & \multicolumn{2}{|c|}{$\operatorname{aSlgAD}$} & \multicolumn{2}{|c|}{ pSIgAD } & $p$ \\
\hline Number & \multicolumn{2}{|c|}{25} & \multicolumn{2}{|c|}{33} & \\
\hline Age in years (mean) & \multicolumn{2}{|c|}{$4-17.5(8.80)$} & \multicolumn{2}{|c|}{$4-17.5(6.82)$} & $0.065^{1}$ \\
\hline Sex male/female & \multicolumn{2}{|c|}{$15 / 10$} & \multicolumn{2}{|c|}{$11 / 22$} & \\
\hline Infections in total & 18 & $72.00 \%$ & 26 & $78.79 \%$ & 0.550 \\
\hline Respiratory tract infections & 16 & $64.00 \%$ & 25 & $75,76 \%$ & 0.330 \\
\hline Lower respiratory tract & 12 & $48.00 \%$ & 15 & $45.45 \%$ & 0.847 \\
\hline Upper respiratory tract & 12 & $48.00 \%$ & 16 & $48.48 \%$ & 0.971 \\
\hline Otitis media & 4 & $16.00 \%$ & 4 & $12.12 \%$ & $0.715^{2}$ \\
\hline Gastrointestinal infections & 0 & $0 \%$ & 2 & $6.06 \%$ & $0.501^{2}$ \\
\hline Urinary tract infections & 2 & $8.00 \%$ & 4 & $12.12 \%$ & $0.690^{2}$ \\
\hline Deep-seated infections & $2^{3}$ & $8.00 \%$ & $1^{3}$ & $3.03 \%$ & $0.572^{2}$ \\
\hline Allergy in total & 13 & $52.00 \%$ & 23 & $69.70 \%$ & 0.169 \\
\hline Food allergy & 4 & $16.00 \%$ & 6 & $18.18 \%$ & $1.000^{2}$ \\
\hline Atopic dermatitis & 2 & $8.00 \%$ & 4 & $12.12 \%$ & $0.690^{2}$ \\
\hline Asthma & 6 & $24.00 \%$ & 13 & $39.39 \%$ & 0.216 \\
\hline Allergic rhinitis & 5 & $20.00 \%$ & 9 & $27.27 \%$ & 0.522 \\
\hline Urticaria & 0 & $0 \%$ & 3 & $9.09 \%$ & $0.251^{2}$ \\
\hline Autoimmunity in total & 11 & $44.00 \%$ & 4 & $12.12 \%$ & 0.006 \\
\hline Diabetes type I & 3 & $12.00 \%$ & 1 & $3.03 \%$ & $0.305^{2}$ \\
\hline Juvenile idiopathic arthritis/recurrent arthritis & 3 & $12.00 \%$ & 1 & $3.03 \%$ & $0.305^{2}$ \\
\hline Celiac disease & 2 & $8.00 \%$ & 2 & $6.06 \%$ & $1.000^{2}$ \\
\hline Thyroiditis & 1 & $4.00 \%$ & 2 & $6.06 \%$ & $1.000^{2}$ \\
\hline Vitiligo & 1 & $4.00 \%$ & 0 & $0 \%$ & $0.431^{2}$ \\
\hline Inflammatory bowel disease & 1 & $4.00 \%$ & 0 & $0 \%$ & $0.431^{2}$ \\
\hline Malignancies & 1 & $4.00 \%$ & 1 & $3.03 \%$ & $1.000^{2}$ \\
\hline Family history & $1^{4}$ & $4.00 \%$ & $2^{4}$ & $6.06 \%$ & $1.000^{2}$ \\
\hline
\end{tabular}

aSIgAD - absolute selective IgA deficiency, pSIgAD - partial selective IgA deficiency, $p$ was calculated using Pearson's $\chi^{2}$ test and in marked cases using 'Shapiro-Wilk test, ${ }^{2}$ Fischer's exact test, ${ }^{3}$ sepsis, , ${ }^{4}$ common variable immunodeficiency

compensatively; however, it does not completely replace $\operatorname{IgA}$ functionally, particularly in the respiratory tract [14, 15]. Secretory IgA in the mucosa neutralises infectious agents or antigens and interferes with growth factors and enzymes, impairing pathogen growth and invasion and induces a loss of bacterial plasmids connected with antibiotic resistance [16]. According to the literature, recurrent sinopulmonary infections were the dominant manifestation, concerning $40-90 \%$ of patients at the time of diagnosis of SIgAD [15], which is in line with our results. In the group we studied, the level of deficiency did not affect the observed frequency of respiratory tract infections, similar results were obtained by Moschese et al. [5]. Analysis of parental questionnaires carried out by Janzi et al. [3] indicated an increased risk of pseudocroup at year 1 in the group of aSIgAD compared to pSIgAD and children with normal IgA serum; there were no significant differences in the occurrence of pneumonia, bronchitis, otitis media, and RSV-infections in early childhood. The results of one prospective study showed that children with SIgAD were at increased risk for a higher number of respiratory infections, resulting in significantly lower pulmonary function, and the incidence was related to the variant of SIgAD [17].

Gastrointestinal infections, including those caused by Giardia lamblia, as well as urinary tract infections, are also reported in SIgAD, but probably due to the existence of compensatory mechanisms their percentage is not high [5, 18-20]. In our study, the incidence of gastrointestinal and urinary tract infection was noticeably lower than the incidence of sinopulmonary conditions and did not differ between the groups. It should also be emphasised that sepsis occurred in both variants of SIgAD but affected a small percentage of children, which is in line with the literature [18].

The impact of recurrent infections on the maturation of the immune system in the first years of life is currently 
underlined. Chronic inflammation induces immunometabolic, immunoendocrine, and microbiota alterations, which invaluably affects health programming and may cause potential health consequences in later life [9, 21].

Allergic diseases appear to be the second most frequent manifestation of SIgAD, and their prevalence ranges, depending on the literature data, from $12.7 \%$ to as many as $80 \%$ of patients; what is more, every fourth diagnosis of the SIgAD is made during evaluation of allergic disorders $[2,15]$. Among the proposed mechanisms causing an increase in the frequency of allergies, the key role of IgA in protecting mucous membranes against the entry of antigens, as well as the possible compensatory increase in the concentration of immunoglobulin $\mathrm{E}$, are emphasised [15]. In our study, allergy was observed in every second patient with aSIgAD and in nearly $70 \%$ of patients with pSIgAD, but the difference was not statistically significant. In both groups, children suffered most often from asthma, allergic rhinitis, and food allergy, which concurs with earlier reports. Previous studies do not provide a definite answer on the risk of occurrence allergy in pSIgAD $[3,5,17]$; however, according to our results, the incidence of individual allergic disorders does not show differences between the variants of deficiency.

Recent reports indicate a high percentage of the concomitance of aSIgAD and autoimmune disorders, ranging between $7 \%$ and 36\% [22]. However, it may be underestimated due to the insufficient awareness of the importance of immunology screening in patients with autoimmunity [23]. The weakening of mucosal protection resulting from SIgAD facilitates the penetration of antigens, which may result in cross-reactions with self-antigens. Occurrence of certain human leukocyte antigen (HLA) genes on the MHC locus of chromosome 6 favours the development of both SIgAD and autoimmune diseases; therefore, some authors even postulate the possible autoimmune aetiology of SIgAD [24]. Other putative mechanisms include defective antigen clearance leading to chronic inflammation or abnormalities in T-cell regulation, especially in regulatory T cells and reduced switched memory B cells $[23,25,26]$. Strong literature data support the association between SIgAD and some autoimmune diseases, including celiac disease, lupus erythematosus, thyroiditis, diabetes type I, inflammatory bowel diseases or juvenile idiopathic arthritis, and other disorders - for example, scleroderma, and autoimmune hepatitis - coexist less often with SIgAD [9, 26]. Autoimmunity in pSIgAD is to date rarely discussed in the literature; Shakkottai et al. [27] noted the co-existence of PSIgAD and rheumatic diseases, and Moschese et al. [5] reported similar prevalence of autoimmune disorders in both variants of SIgAD. Interestingly, our results indicate a statistically higher incidence of autoimmune disorders in aSIgAD than in pSIgAD (40\% vs. 12.12 ,\% in total). Diabetes type I, juvenile idiopathic arthritis, celiac disease, and thyroiditis were recognised the most often - they affected about $3-12 \%$ of patients. In interpreting the results, however, the average age of patients should be taken into account, while most autoimmune diseases manifest themselves in adolescents and adults.

Several reports suggest an increased risk of neoplasia coexisting with SIgAD; nonetheless, it applies to adult patients [28]. In the group we studied, malignancies occurred only in two patients. Positive family history in both groups was present only in a few percent of the patients, and in all cases family members were diagnosed with CVID. According to the literature, familial cases may constitute up to $30 \%$ of patients with IgAD and CVID and are associated with a higher frequency of lower respiratory tract infections than sporadic ones [29].

There are some possible limitations of the study. They result from its retrospective nature and small group sizes corresponding to a low incidence of symptomatic course of the disease. Multicentre and prospective studies on the current topic are therefore recommended for the future.

In conclusion, the findings reported here shed new light on the significance of decreased IgA level. Due to the similar clinical course of both variants of SIgAD, all symptomatic patients should receive specialist care and undergo systematic follow-up visits to assess their general health, with particular emphasis on the respiratory tract, as well as the occurrence of allergic and autoimmune disorders. Moreover, in children with autoimmune diseases, basic immunology screening should be considered.

\section{DISCLOSURE}

The authors declare no conflict of interest.

\section{REFERENCES}

1. Pan-Hammarström Q, Hammarström L. Antibody deficiency diseases. Eur J Immunol 2008; 38: 327-333.

2. Yel L. Selective IgA Deficiency. J Clin Immunol 2010; 30: 10-16.

3. Janzi M, Kull I, Sjöberg R, et al. Selective IgA deficiency in early life: association to infections and allergic diseases during childhood. Clin Immunol Orlando Fla 2009; 133:78-85.

4. Yazdani R, Azizi G, Abolhassani H, et al. Selective IgA Deficiency: Epidemiology, Pathogenesis, Clinical Phenotype, Diagnosis, Prognosis and Management. Scand J Immunol 2017; 85: 3-12.

5. Moschese V, Chini L, Graziani S, et al. Follow-up and outcome of symptomatic partial or absolute IgA deficiency in children. Eur J Pediatr 2019; 178: 51-60.

6. Jesenak M, Banovcin P, Jesenakova B, et al. Pulmonary manifestations of primary immunodeficiency disorders in children. Front Pediatr 2014; 2: 77.

7. Yazdani R, Abolhassani H, Asgardoon M, et al. Infectious and Noninfectious Pulmonary Complications in Patients With Primary Immunodeficiency Disorders. J Investig Allergol Clin Immunol 2017; 27: 213-224.

8. Nurkic J, Numanovic F, Arnautalic L, et al. Diagnostic Significance of Reduced IgA in Children. Med Arch Sarajevo Bosnia Herzeg 2015; 69: 236-239.

9. Koenen MHM, van Montfrans JMJ, Sanders EAME, et al. Immunoglobulin A deficiency in children, an undervalued clinical issue. Clin Immunol Orlando Fla 2019; 209: 108293. 
10. Domínguez O, Giner MT, Alsina L, et al. [Clinical phenotypes associated with selective IgA deficiency: a review of 330 cases and a proposed follow-up protocol]. An Pediatr Barc Spain 2012; 76: 261-267.

11. Aytekin C, Tuygun N, Gokce S, et al. Selective IgA deficiency: clinical and laboratory features of 118 children in Turkey. J Clin Immunol 2012; 32: 961-966.

12. Eşki E, Usta BE, Asilsoy S, et al. Sensorineural Hearing Loss in Selective Immunglobulin A Deficiency. Turk Arch Otorhinolaryngol 2017; 55: 31-33.

13. Ozkan H, Atlihan F, Genel F, et al. IgA and/or IgG subclass deficiency in children with recurrent respiratory infections and its relationship with chronic pulmonary damage. J Investig Allergol Clin Immunol 2005; 15: 69-74.

14. Brandtzaeg P, Karlsson G, Hansson G, et al. The clinical condition of IgA-deficient patients is related to the proportion of IgD- and IgM-producing cells in their nasal mucosa. Clin Exp Immunol 1987; 67: 626-636.

15. Yazdani R, Latif A, Tabassomi F, et al. Clinical phenotype classification for selective immunoglobulin A deficiency. Expert Rev Clin Immunol 2015; 11: 1245-1254.

16. Pilette C, Ouadrhiri Y, Godding V, et al. Lung mucosal immunity: immunoglobulin-A revisited. Eur Respir J 2001; 18: 571-588.

17. Živković J, Lipej M, Banić I, et al. Respiratory and allergic disorders in children with severe and partial Immunoglobulin A immunodeficiency. Scand J Immunol 2019; 14: e12828.

18. Ludvigsson JF, Neovius M, Hammarström L. Risk of Infections Among 2100 Individuals with IgA Deficiency: a Nationwide Cohort Study. J Clin Immunol 2016; 36: 134-140.

19. Lougaris V, Sorlini A, Monfredini C, et al. Clinical and Laboratory Features of 184 Italian Pediatric Patients Affected with Selective IgA Deficiency (SIgAD): a Longitudinal Single-Center Study. J Clin Immunol 2019; 39: 470-475.

20. Brandtzaeg P. Update on mucosal immunoglobulin A in gastrointestinal disease. Curr Opin Gastroenterol 2010; 26: 554-563.

21. Pabst O, Slack E. IgA and the intestinal microbiota: the importance of being specific. Mucosal Immunol 2020; 13: 12-21.

22. Etzioni A. Immune deficiency and autoimmunity. Autoimmun Rev 2003; 2: 364-369.

23. Abolhassani H, Gharib B, Shahinpour S, et al. Autoimmunity in patients with selective IgA deficiency. J Investig Allergol Clin Immunol 2015; 25: 112-119.

24. Wang N, Hammarström L. IgA deficiency: what is new? Curr Opin Allergy Clin Immunol 2012; 12: 602-608.

25. Jacob CMA, Pastorino AC, Fahl K, et al. Autoimmunity in IgA deficiency: revisiting the role of IgA as a silent housekeeper. J Clin Immunol 2008; 28 (Suppl 1): S56-61.

26. Odineal DD, Gershwin ME. The Epidemiology and Clinical Manifestations of Autoimmunity in Selective IgA Deficiency. Clin Rev Allergy Immunol 2020; 58: 107-133.

27. Shakkottai A, Bupathi K, Patel AP, et al. Children with partial IgA deficiency: clinical characteristics observed in the pediatric rheumatology clinic. Clin Pediatr (Phila) 2012; 51: 46-50.

28. Ludvigsson JF, Neovius M, Ye W, et al. IgA deficiency and risk of cancer: a population-based matched cohort study. J Clin Immunol 2015; 35: 182-188.

29. Karaca NE, Severcan EU, Bilgin BG, et al. Familial inheritance and screening of first-degree relatives in common variable immunodeficiency and immunoglobulin A deficiency patients. Int J Immunopathol Pharmacol 2018; 32: 2058738418779458. 